\title{
RATIONAL SOLUTIONS OF LINEAR DIFFERENTIAL EQUATIONS
}

\author{
J.-P. BEZIVIN and P. ROBBA
}

(Received 24 February 1987)

Communicated by J. H. Loxton

\begin{abstract}
Let $L$ be a linear differential operator with rational coefficients such that 0 is not an irregular singularity of $L$ and that for sufficiently many $p$ 's the equation $L v=0$ has no zero solution mod $p$. We show that if $u$ is a formal power series whose coefficients are p-adic integers for almost all $p$ and if $L u$ is rational, then $u$ too is rational.
\end{abstract}

1980 Mathematics subject classification (Amer. Math. Soc.) (1985 Revision): 12 H 25, 11 B 37.

\section{Statement of the results and examples}

1.1. Let $L$ be a linear differential operator with rational coefficients and let $u$ be a formal power series such that $L u$ is rational. We ask whether $u$ too is rational. We first review the known results on this topic.

1.2. The first mathematician interested by this problem was G. Pólya who proved the following result in 1921.

THEOREM [8]. Let $u=\sum_{n \geq 0} u_{n} x^{n} \in \mathbf{Z}[[x]]$ be a formal power series with integral coefficients. If its derivative $u^{\prime}=\sum_{n \geq 1} n u_{n} x^{n-1}$ is a rational function, then $u$ too is rational

1.3. This result was extended in 1965 by D. Cantor.

(C) 1989 Australian Mathematical Society 0263-6115/89 \$A2.00+0.00 
THEOREM [3]. Let $u=\sum_{n \geq 0} u_{n} x^{n}$ be a formal power series whose coefficients are algebraic integers and let $P$ be a non zero polynomial with algebraic coefficients. If the series $\sum_{n \geq 0} P(n) u_{n} x^{n}$ is rational then $u$ too is rational.

1.4. The hypothesis that the coefficients are algebraic integers is in some way necessary as shown by the counterexample $u=\sum x^{n} / n=-\log (1-x)$ which is not rational, while $u^{\prime}=\sum x^{n}=1 /(1-x)$ is rational.

Of course it is well known that this condition can be weakened. Let $K$ be an algebraic number field and assume that the coefficients $u_{n}$ of $u$ belong to $K$. Then the conclusions of the two preceding theorems still hold even if we only assume that $u$ satisfies the condition:

(*) for all but a finite number of prime ideals $\mathfrak{p}$ of $K$, all the coefficients $u_{n}$ of $u$ are $\mathfrak{p}$-adic integers.

If $u \in K((x))$ (resp. $u \in K[[x]]$ ) satisfies (*), we shall write from now on that $u \in \tilde{K}((x))$ (resp. $u \in \tilde{K}[[x]])$.

It is easily seen that $\tilde{K}((x))$ is a subfield of $K((x))$ stable under the action of the derivation. It is clear that $K[x]$ is contained in $\tilde{K}((x))$ and therefore $K(x)$ is also contained in $\tilde{K}((x))$. This shows that if $u$ does not satisfy the condition $(*), u$ is not rational.

1.5. Recently J.-P. Bezivin [2] proposed to interpret Cantor's result in terms of differential operators: if $P \in K[Y]$, consider the Euler differential operator $L=P(x D)$ where $D$ stands for $d / d x$; then $\sum_{n} P(n) u_{n} x^{n}=L u$. He also gave a new class of differential operators with a property similar to that of Euler operators.

Before stating Bezivin's result, we give a new definition. We shall say that the differential operator $L=\sum A_{i} D^{i} \in K(x)[D]$ is a Pólya operator at 0 if, for every $u \in \tilde{K}((x)), L u$ rational implies that $u$ is rational (thus Cantor's result can be reformulated: every Euler differential operator $L=P(x D)$, with $P \in K[Y]$, is a Pólya operator at 0 ). To save space, in what follows, when we say "Pólya operator", we mean "P6lya operator at 0".

An easy consequence of this definition is that if $L_{1}$ and $L_{2}$ are Polya operators, then $L_{1} L_{2}$ is a Pólya operator; also if $L_{1} L_{2}$ is a Pólya operator then $L_{2}$ is a Pólya operator ([2], Proposition 1). Clearly differential operators of order 0 are Pólya operators. Therefore, for any rational functions $Q, R \in K(x)$, the differential operators $L$ and R.L. $Q$ are either simultaneously Pólya operators or simultaneously not Pólya operators. We shall say that $L$ and R.L. $Q$ are equivalent differential operators.

1.6. We now exhibit Bezivin's new class of Pólya operators. 
THEOREM [2]. Let $L=\sum_{k=0}^{s} A_{k} D^{k} \in K[x][D]$ where $A_{0}$ is a non zero constant and for all $k \geq 1$ the degree of the polynomial $A_{k}$ is $\leq k-1$. Assume further that 0 is not an irregular singular point for $L$. Then $L$ is a Pólya operator.

A special case of this situation is the case of differential operators with constant coefficients $L=P(D)$ with $P \in K[Y]$ and $P(0) \neq 0$. We can combine this result with Pólya's result to conclude that every operator $L=P(D) \in K[D]$ is a Pólya operator (indeed we can write $L=Q(D) D^{s}$ with $Q \in K[Y], Q(0) \neq 0$, and $s$ an integer $\geq 0$ ).

1.7. The main result. For each prime ideal $\mathfrak{p}$ of $K$ we denote by $\bar{K}_{\mathfrak{p}}$ the associated residue field. Also $p$ will denote the prime number divided by $\mathfrak{p}$. If $L \in K(x)[D]$ we denote $L_{\mathfrak{p}} \in \bar{K}_{\mathfrak{p}}(x)[D]$ its reduction modulo $\mathfrak{p}$ when this reduction is defined (which is the case for all but a finite number of prime ideals $\mathfrak{p})$.

ThEOREM. Let $L \in K(x)[D]$. Assume that

(a) 0 is not an irregular singularity for $L$,

(b) there exists an infinite set $S$ of prime ideals with

$$
\sum_{p \in S, \mathfrak{p} \mid p} \frac{1}{p} \log p=+\infty
$$

such that for $\mathfrak{p} \in S$ the reduced equation $L_{\mathfrak{p}} v=0$ has no non zero solution in $\bar{K}_{\mathfrak{p}}((x))$.

Then $L$ is a Pólya operator.

We recall (see [6] for example) that the equation $L_{\mathfrak{p}} v=0$ has a non zero solution in $\bar{K}_{\mathfrak{p}}((x))$ if and only if it has a non zero solution in $\bar{K}_{\mathfrak{p}}(x)$.

We observe that the condition (1.7.1) is verified if $S$ has positive Dirichlet density (see [7], page 131 for the definition). In particular this condition is satisfied if $S$ contains all but a finite number of the prime ideals.

We shall now discuss the necessity of condition (a) and give examples of differential operators satisfying condition (b).

1.8. Singular points. Let $L=\sum A_{i} D^{i} \in K(x)[D]$ of order $l$ and let $a \in K^{\text {alg }}$ (algebraic closure of $K$ ). By Fuchs' condition $a$ is an ordinary point or a regular singular point for $L$ if

$$
\operatorname{ord}_{a} A_{l}-l=\min _{i}\left(\operatorname{ord}_{a} A_{i}-i\right)
$$

(for $a=\infty$ the condition reads $\operatorname{ord}_{\infty} A_{l}+l=\min _{i}\left(\operatorname{ord}_{\infty} A_{i}+i\right)$ ). We shall say that $a$ is a totally irregular singular point for $L$ if

$$
\operatorname{ord}_{a} A_{0}<\min _{i \geq 1}\left(\operatorname{ord}_{a} A_{i}-i\right)
$$

(for $a=\infty$ the condition reads ord $A_{\infty}<\min _{i \geq 1}\left(\operatorname{ord}_{\infty} A_{i}+i\right)$ ). 
1.9. PROPOSITION ([2], Corollaire 1). If 0 is a totally irregular singular point for $L, L$ is not a Pólya operator.

For an operator of order 1, an irregular singular point is a totally irregular singular point.

This situation is illustrated by the following example: $L=x^{2} D+x-1$, $u=\sum_{n \geq 0} n ! x^{n} \in \mathbf{Z}[[x]]$ satisfies $L u=-1$ but $u \notin \mathbf{Q}(x)$.

Observe that by Proposition 1.11 below, if 0 is a totally irregular singular point for $L, L$ satisfies the condition (b) of Theorem 1.7.

If 0 is an irregular singular point for $L$ but not a totally irregular singular point, we do not know whether or not $L$ is a Pólya operator.

1.10. Assume that the equation $L v=0$ has a non zero solution $v \in \tilde{K}((x))$. Then for almost all prime ideals $\mathfrak{p}$ we obtain by reduction mod $\mathfrak{p}$ a non zero solution of $L_{\mathfrak{p}} v=0$, and therefore the condition (b) of Theorem 1.5 is not satisfied.

Of course if this solution $v$ is not rational, $L$ is not a Pólya operator. For example, consider $L=(1-2 x) D+1 ; u=\sqrt{1-2 x} \in \mathbf{Z}[[x]]$ is solution of $L u=0$ but $u \notin Q(x)$. Again consider $L=x(1-x) D^{2}+(1-2 x) D-1 / 4$; $u=F(1 / 2,1 / 2,1 ; x) \in \mathbf{Z}[1 / 2][[x]] \subset \tilde{\mathbf{Q}}[[x]]$ is solution of $L u=0$ but $u \notin \mathbf{Q}(x)$.

If $L v=0$ has a rational solution $v$, then we have the decomposition $L=$ $L_{1} \circ\left(D-v^{\prime} / v\right)$ and $D-v^{\prime} / v=v \circ D \circ v^{-1}$, equivalent to $D$, is a Pólya operator. The problem is reduced to seeing if $L_{1}$ is a Pólya operator.

It may happen that condition (b) of Theorem 1.7 is not satisfied even if $L v=0$ has no non zero solution in $\tilde{K}((x))$. This is the case if $L=x D-\alpha$ with $\alpha \in \mathbb{Q}-\mathbf{Z}$ : indeed for every $\mathfrak{p}$ which does not divide the denominator of $\alpha$, there exists an integer $\alpha(\mathfrak{p})$ such that $\alpha \equiv \alpha(\mathfrak{p}) \bmod \mathfrak{p}$ and $v=x^{\alpha(\mathfrak{p})}$ is a solution of $L_{\mathfrak{p}} v=0$.

We shall now give examples of differential operators which satisfy condition (b).

1.11. PROPOSITION. If $L$ possesses a totally irregular singular point, then for all but a finite number of prime ideals $\mathfrak{p}$ the reduced equation $L_{p} v=0$ has no non zero solution in $\bar{K}_{\mathfrak{p}}((x))$.

The hypothesis of Theorem 1.6 implies that $\infty$ is a totally irregular singular point for $L$. Thus Bezivin's class of Pólya operators is covered by our criterion.

1.12. We give another example, involving Euler differential operators, where condition (b) of Theorem 1.7 is satisfied. Consider $L=x D-\alpha$ with $\alpha$ irrational. Then by Tchebotarev's Density Theorem ([7], Theorem 10.4), there exists a set $S$ of prime ideals of positive density such that for all $\mathfrak{p} \in S \alpha$ is not congruent 
to an integer modulo $\mathfrak{p}$ and thus $L_{\mathfrak{p}} v=0$ has no non zero solution in $\bar{K}_{\mathfrak{p}}((x))$, and $L$ is a Pólya operator as 0 is a regular singular point for $L$.

Although the class of Pólya operators is stable under composition (1.5) the class of operators satisfying condition (b) of Theorem 1.7 is not stable under composition. Indeed for all prime numbers $p$ at least one of the three numbers 2 , 3,6 is a quadratic residue modulo $p$; therefore if $L=(x D-\sqrt{2})(x D-\sqrt{3})(x D-$ $\sqrt{6}$ ) then for all prime ideals $\mathfrak{p}$ of $K=\mathbf{Q}(\sqrt{2}, \sqrt{3}), L_{\mathrm{p}} v=0$ has a solution $\bmod \mathfrak{p}$ in $\bar{K}_{\mathrm{p}}(x)$, while each of the three factors satisfy condition (b). Of course we see that $L$ is a Pólya operator as each of the three factors is a Polya operator.

1.13. For future applications to transcendental results (see for example ' $A$ new $p$-adic method for proving irrationality and transcendence results' by the same authors, to appear) we would like to mention that the proof of Theorem 1.7 implies the following result:

ThEOREM. Let $L \in K[x][D]$ and $u \in K[[x]]$. Assume that

(i) for any infinite place of $K$ (that is, for any embedding of $K$ in $C$ ), $u$ has a non zero radius of convergence,

(ii) $u$ satisfies condition (*),

(iii) there exists an infinite set $S$ of prime ideals with

$$
\sum_{p \in S, p \mid p} \frac{1}{p} \log p=+\infty
$$

such that for all $\mathfrak{p} \in S$ the reduced equation $L_{\mathfrak{p}} v=0$ has no non zero solution in $\bar{K}_{\mathfrak{p}}(x)$,

(iv) $L u \in K(x)$.

Then $u \in K(x)$.

Indeed in the proof of Theorem 1.7, the hypothesis that 0 is not an irregular singularity for $L$ is used only to show that $u$ has a non zero radius of convergence for any embedding of $K$ in C (see point (ii) of paragraph 2.6).

1.14. In paragraph 2, we give the proofs of Theorem 1.7 and Proposition 1.11. In paragraph 3 we discuss in detail the case of operators of order 1 where we have a good, albeit incomplete, understanding of the situation.

This article is a development of the ideas of [2] and [10].

\section{Proofs of the results}

2.1. We begin with the proof of Proposition 1.11, which is simpler. 
Proof of Proposition 1.11. Let $L=\sum_{i=0}^{l} A_{i} D^{i} \in K(x)[D]$, of order $l$, and assume that $a \in K^{\text {alg }}$ is a totally irregular singular point for $L$. By extending $K$ if necessary we may assume that $a \in K$.

Consider a prime ideal $\mathfrak{p}$ of $K$ such that the reduction $L_{\mathfrak{p}}$ of $L$ is defined, $a$ is a p-adic integer with reduction $\bar{a}_{\mathfrak{p}} \in \bar{K}_{\mathfrak{p}}$, and $\operatorname{ord}_{a} A_{0}=\operatorname{ord}_{\bar{a}_{\mathfrak{p}}} \bar{A}_{0 \mathrm{p}}$. These conditions are verified for all but a finite number of prime ideals $\mathfrak{p}$. For such a $\mathfrak{p}, \bar{a}_{\mathfrak{p}}$ is a totally irregular singular point for $L_{\mathfrak{p}}$ as

$$
\operatorname{ord}_{a_{\mathfrak{p}}} \bar{A}_{0 \mathrm{p}}=\operatorname{ord}_{a} A_{0}<\min \left(\operatorname{ord}_{a} A_{i}-i\right) \leq \min _{i}\left(\operatorname{ord}_{\bar{a}_{\mathrm{p}}} \bar{A}_{i \mathrm{p}}-i\right) .
$$

Let $v \in \bar{K}_{\mathfrak{p}}(x), v \neq 0$. It follows from (2.1.1) that

$$
\operatorname{ord}_{\bar{a}_{\mathfrak{p}}} L_{\mathfrak{p}} v=\operatorname{ord}_{\bar{a}_{\mathfrak{p}}} \bar{A}_{0 \mathfrak{p}}+\operatorname{ord}_{\bar{a}_{\mathfrak{p}}} v
$$

and thus $L_{\mathfrak{p}} v$ cannot be zero if $v$ is not zero.

2.2. In order to prove that the solution $u$ of our differential equation is rational we shall need the following criterion of rationality applied to $u(1 / x)$.

For every prime ideal $\mathfrak{p}$ of $K, K$ will be equipped with the associated normalized absolute value $\|_{\mathfrak{p}}$ (see [1], page 40 ) and $C_{\mathfrak{p}}$ will denote an algebraically closed extension of $K$ complete under a valuation extending that of $K$. We denote $\mathscr{P}(K)$ the set of prime ideals of $K$.

For every infinite place $w$ of $K, K$ can be embedded in $C$. We denote $N(w)$ the degree of $C$ over the completion of $K, N(w)=1$ or 2 . We denote $I(K)$ the set of infinite places of $K$.

LEMMA (Pólya-Bertrandias, [1], Théorème 5.4.6). Let $K$ be an algebraic number field and $f=\sum_{n \geq 0} a_{n} / x^{n} \in K[[1 / x]]$. Assume that there exists a finite subset $\mathscr{P}_{1}(K)$ of $\mathscr{P}(K)$ such that

(i) for all $\mathfrak{p} \notin \mathscr{P}_{1}(K)$ and for all $n,\left|a_{n}\right|_{p} \leq 1$,

(ii) for all $w \in I(K), f$ defines in $\mathbf{C}$ a function analytically extendable on a connected domain $B_{w}$ whose complement is bounded and has transfinite diameter $d_{w}$,

(iii) for $\mathfrak{p} \in \mathscr{P}_{1}(K), f$ defines in $C_{\mathfrak{p}}$ a function extendable by an analytic element on a set $B_{\mathfrak{p}}$ of $C_{\mathfrak{p}}$ whose complement is bounded and of transfinite diameter $d_{\mathfrak{p}}$,

(iv) $\left(\prod_{w \in I(K)} d_{w}^{N(w)}\right) \times\left(\prod_{p \in \mathscr{P}_{1}(K)} d_{\mathfrak{p}}\right)<1$.

Then $f$ is rational.

We will not recall the definition of the transfinite diameter, but we observe that in our application the complement of the set $B_{\mathfrak{p}}$ will have the form $\bigcup_{1 \leq i \leq s} B^{-}\left(c_{i}, r\right)$, where $B^{-}\left(c_{i}, r\right)=\left\{x \in C_{p} ;\left|x-c_{i}\right|<r\right\}$, the $c_{i}$ are in different residue classes, and $r<1$. In this case it follows from [1], Example 5.4.4, that the transfinite diameter of this set is $r^{1 / s}$. 
We recall that an analytic element on $B_{\mathfrak{p}}$ is the uniform limit on $B_{\mathfrak{p}}$ of a sequence of rational functions without poles in $B_{\mathfrak{p}}$. If $B_{\mathfrak{p}}$ has the form described above and $u$ is an analytic element on $B_{\mathfrak{p}}$ which is zero in a neighborhood of a point $a$ of $B_{\mathfrak{p}}$, then $u$ is zero everywhere in $B_{\mathfrak{p}}$. Thus if $f$ is defined in a neighborhood of $a$ by a power series and $f$ can be extended into an analytic element on $B_{\mathfrak{p}}$, then this extension is unique.

2.3. LemMA ([10], Theorem 2.10). Let $C$ be a complete ultrametric valued field, and assume that its residue field has characteristic $p \neq 0$. Let $L \in C[x][D]$. Let $\Delta$ be the disk $B^{+}(c, r)=\{x \in C ;|x-c| \leq r\}$, and consider $L$ as an endomorphism of $H(\Delta)$, the space of analytic elements on $\Delta$. If $L$ is surjective, then $L$ is injective.

For the reader's convenience we outline the proof.

Proof. Assume that $L$ is not injective in $H(\Delta)$ and let $u \in H(\Delta)$ such that $L u=0$. Choose $a_{m} \in C$ with $r^{m} \leq\left|a_{m}\right| \leq \sigma r^{m}, \sigma>1$, belonging to the value group of $C$. The functions

$$
u_{k}=\sum_{n \geq 0} a_{p^{2 k n}} \frac{(x-c)^{p^{2 k n}}}{p^{k n}}
$$

are unbounded in $\Delta$, and further the functions $u u_{k}$ are linearly independent over $H(\Delta)$. On the other hand one sees that $L\left(u u_{k}\right) \in H(\Delta)$. This implies that $L$ is not surjective.

2.4. We shall need some properties of differential operators in characteristic $p \neq 0$.

LEMMA. let $k$ be a field of characteristic $p \neq 0$. Let $L \in k[x][D]$. If the equation $L v=0$ has no non zero solutions in $k((x))$, there exist $P, Q \in K[x][D]$ and $\pi \in k[x], P \neq 0, \pi \neq 0$, such that

$$
P L+Q D^{p}=\pi .
$$

Further there exists a constant $\sigma$ depending only on the degrees of the coefficients of $L$ and not on $p$, such that $P, Q$ and $\pi$ can be assumed to satisfy the additional conditions: ord $P \leq p-1$, ord $Q \leq$ ord $L-1$, the degrees of $\pi$ and the degrees of the coefficients of $P$ and $Q$ are at most $\sigma p$.

If $L=\sum A_{i} D^{i}$ and if we define $N(L)=\max _{i}\left(\operatorname{deg} A_{i}\right)$, then the proof of the lemma shows that one can take $\sigma=N(L)$. The constant $\sigma=N(L)$ seems to be optimal.

ProOF. The first part of the lemma is a direct consequence of [5], Corollary 6.1.2.2 which asserts that $\operatorname{Ker} L=\{0\}$ if and only if the left sided ideal generated 
by $L$ and $D^{p}$ in $k(x)[D]$ is $k(x)[D]$. So assume that we have $P, Q$, and $\pi$ satisfying (2.4.1), we shall construct new differential operators $P_{1}, Q_{1}$ and a polynomial $\pi_{1} \neq 0$ satisfying (2.4.1) and the additional conditions.

Condition on the orders. (a) Observe that as $k$ has characteristic $p$, one has $D^{p} L=L D^{p}$. If we set $P=P_{1}+S D^{p}$ with ord $P_{1} \leq p-1$ and $Q_{1}=Q+S L$ then we have $P_{1} L+Q_{1} D^{p}=\pi$ and $\operatorname{ord} Q_{1} \leq \operatorname{ord}\left(P_{1} L\right)-p \leq \operatorname{ord} L-1$. So we may assume that $P, Q$ satisfy (2.4.1), ord $P \leq p-1$, ord $Q \leq \operatorname{ord} L-1$.

Conditions on the degrees of the coefficients. (b) Let $U$ be the vector space of differential polynomials with rational coefficients, of order at most $p-1$, thus $U \simeq[k(x)]^{p}$. We consider the following linear forms $\mathscr{L}_{j}$ on $U:$ for $R \in U$

$$
\mathscr{L}_{j}(R)=\text { coefficient of } D^{j} \text { in } R L, \quad 0 \leq j \leq p-1 .
$$

If $R=\sum_{i} R_{i} D^{i}$, one has $\mathscr{L}_{j}(R)=\sum_{i=0}^{p-1} a_{j i} R_{i}$ where the $a_{j i}$ are expressed in term of the derivatives of the coefficients of $L$ and therefore $a_{j i} \in k[x]$ with $\operatorname{deg}\left(a_{j i}\right) \leq N(L)$.

If $P \in U$ is such that $\mathscr{L}_{0}(P) \neq 0$ and $\mathscr{L}_{j}(P)=0,1 \leq j \leq p-1$, then it is clear that there exists $Q \in k(x)[D], \operatorname{deg} Q \leq \operatorname{deg} L-1$ and $\pi \in k(x), \pi \neq 0$, such that (2.4.1) is satisfied.

Let $r$ be the rank (over $k(x)$ ) of the linear forms $\mathscr{L}_{j}, 1 \leq j \leq p-1$. Then one can find a subset $\mathscr{P}=\left\{j_{1}, \ldots, j_{p-r}\right\} \subset\{0, \ldots, p-1\}$ such that the system $\mathscr{L}_{j}(R)=0,1 \leq j \leq p-1$ determines uniquely the $r$ unknowns $R_{i}, i \notin \mathscr{P}$, in terms of the $p-r$ unknowns $R_{j}, j \in \mathscr{P}$, which can be chosen freely. We obtain

$$
R_{i}=\sum_{j \in \mathscr{P}} \frac{b_{i j}}{\Delta} R_{j}, \quad i \notin \mathscr{P},
$$

where the $b_{i j}$ and $\Delta$ are determinants of rank $r$ extracted from the matrix $\left(a_{j i}\right)$ and therefore polynomials of degrees at most $r N(L)$.

if we substitute these expressions of $R_{i}$ in $\mathscr{L}_{0}(R)$ we obtain

$$
\mathscr{L}_{0}(R)=\sum_{j \in \mathscr{P}} \frac{c_{j}}{\Delta} R_{j}
$$

where the $c_{j}$ are polynomials with $\operatorname{deg}\left(c_{j}\right) \leq(r+1) N(L)$.

By (a) we know that there exists $P \in U$ with $\mathscr{L}_{0}(P) \neq 0, \mathscr{L}_{j}(P)=0$, $1 \leq j \leq p-1$. Therefore the coefficients $c_{j}$ are not all zero. Assume that $c_{k} \neq 0$. Then if we choose $R_{k}=\Delta, R_{j}=0, j \neq k, j \in \mathscr{P}, R_{i}=b_{i k}, i \notin \mathscr{P}$, the coefficients of $R$ have degrees at most $r N(L)$. And we have $R L+Q_{1} D^{p}=\pi_{1}$ with $\operatorname{deg} \pi_{1} \leq(r+1) N(L)$, deg (coefficients of $\left.Q_{1}\right) \leq(r+1) N(L)$. As $r \leq p-1$, this ends the proof of the lemma with $\sigma=N(L)$.

2.5. We recall a result on the analytic extension of solutions of differential equations with polynomial coefficients. Here $C$ denotes an algebraically closed, complete ultrametric valued field. 
For $L=\sum A_{i} D^{i} \in C[x][D]$ we define

$$
m(L):=\max _{i}\left(\operatorname{deg} A_{i}-i\right) .
$$

LEMMA ([9]). Let $u$ be an analytic element on the unit open ball of $C$ and $L \in C[x][D]$. Assume that $L u$ is a polynomial. Then $u$ extends into an analytic element on all the residue classes of $C$ except in at most $\operatorname{ord} L+m(L)$ of them.

For the convenience of the reader we outline the proof.

ProOF . (i) Consider the Mittag-Leffler decomposition of $u$

$$
u=u_{\infty}+\sum_{\alpha} u_{\alpha}
$$

where $\alpha$ runs through the residue classes of $C, u_{\infty}$ is an analytic element on the closed unit ball, $u_{\alpha}$ is an analytic element outside the residue class $\alpha$ and tends to zero at infinity.

If $m(L)=0$, then $L u_{\alpha}$ is analytic outside $\alpha$ and tends to zero at infinity. The unicity of the Mittag-Leffler decomposition together with the relation

$$
L u=L u_{\infty}+\sum_{\alpha} L u_{\alpha}
$$

shows that $L u_{\alpha}=(L u)_{\alpha}$ is the singular part of $L u$ associated to $\alpha$ and therefore $L u_{\alpha}=0$, as $L u$ is a polynomial.

But then, as the dimension of the kernel of $L$ is at most the order of $L$, and as the $u_{\alpha}$ are linearly independent, we conclude that there is at most ord $L$ residue classes $\alpha$ with $u_{\alpha} \neq 0$. This proves the lemma in the case $m(L)=0$.

(ii) If $m(L)=m>0$, define $L_{1}=D^{m} L$. Then $m\left(L_{1}\right)=0$, ord $L_{1}=$ ord $L+m(L), L_{1} u$ is a polynomial, and we can apply (i).

(iii) If $m(L)=-m<0$, then necessarily $L=L_{1} D^{m}$ with $L_{1} \in C[x][D]$ and $m\left(L_{1}\right)=0$, ord $L_{1}=\operatorname{ord} L+m(L)$. We apply (i) to $D^{m} u$ and conclude that $u$, as $D^{m} u$, extends in all residue classes except at most ord $L_{1}$ of them.

2.6. ProOF OF THEOREM 1.7. Let $L \in K(x)[D], u \in \tilde{K}((x))$ such that $L u \in K(x)$.

We can find an integer $s \geq 0$ and a polynomial $A \in K[x]$ such that $u=x^{s} u_{1}$ with $u_{1} \in \tilde{K}[[x]], A L$ has polynomial coefficients and $A L u$ is a polynomial. Then the operators $L$ and $L_{1}=A L x^{s}$ are equivalent, $L_{1} \in K[x][D], L_{1} u_{1} \in K[x]$. Further $L$ and $L_{1}$ satisfy simultaneously the conditions (a) and (b) of Theorem 1.7 .

Therefore we can assume without loss of generality that $u \in \tilde{K}[[x]], L \in$ $K[x][D]$ and $L u=\varphi \in K[x], L$ satisfying conditions (a) and (b) of Theorem 1.7. Define $l:=\operatorname{ord} L$. 
Define $f(x):=u(1 / x)=\sum_{n \geq 0} u_{n} / x^{n}$.

(i) The hypothesis $u \in \tilde{K}[[x]]$ means that there exists a finite set $P(K)$ of prime ideals of $K$ such that for all $\mathfrak{p} \notin P(K)$ and all $n,\left|u_{n}\right|_{\mathfrak{p}} \leq 1$.

(ii) For any infinite place $w$ of $K$, we have an embedding of $K$ in $\mathbb{C}$. As 0 is not an irregular singular point of $L$, it is well known that formal power series solution of $L$ have a non zero radius of convergence. Therefore $u$ converges in a disk of positive radius and $f$ defines an analytic function outside a bounded disk of transfinite diameter $d_{w}<+\infty$.

(iii) For any prime ideal $\mathfrak{p} \in P(K)$, let $C_{\mathfrak{p}}$ be an algebraically closed complete extension of $\left(K, \|_{\mathfrak{p}}\right)$.

The exponents of $L$ at 0 , that is, the roots of the indicial polynomial of $L$ at 0 , are algebraic numbers, therefore they are not $p$-adic Liouville numbers and by a result of Clark [4], formal power series solutions of $L$ have a non zero radius of convergence. Therefore, again, $f$ defines in $C_{\mathfrak{p}}$ an analytic function outside a bounded disk of transfinite diameter $d_{\mathfrak{p}}<+\infty$.

(iv) Consider now a prime ideal $\mathfrak{p} \notin P(K)$ such that $L_{\mathfrak{p}}$ is defined and the equation $L_{\mathfrak{p}} v=0$ has no non zero solutions in $\bar{K}_{\mathfrak{p}}((x))$. Then, by Lemma 2.4, we can find $P^{*}, Q^{*} \in \bar{K}_{\mathfrak{p}}[x][D]$ and $\pi^{*} \in \bar{K}_{\mathfrak{p}}[x]$ such that

$$
P^{*} L_{\mathfrak{p}}+Q^{*} D^{p}=\pi^{*} \text {. }
$$

We may assume that ord $P^{*} \leq p-1$, ord $Q^{*} \leq$ ord $L_{\mathfrak{p}}-1$ and that the degrees of $\pi^{*}$ and of the coefficients of $P^{*}$ and $Q^{*}$ are bounded by $\sigma_{1} p$, where $\sigma_{1}$ is a constant depending only on $L$ and not on $p=\operatorname{char} \bar{K}_{\mathfrak{p}}$.

Again $C_{\mathrm{p}}$ denotes an algebraically closed complete valued extension of $\left(K, \|_{\mathrm{p}}\right)$. Consider liftings $P, Q \in C_{\mathfrak{p}}[x][D]$ and $\pi \in C_{\mathfrak{p}}[x]$ of $P^{*}, Q^{*}$ and $\pi^{*}$ which preserve the orders and the degrees of the polynomials. We may also assume that in each residue class $\pi$ has at most one zero, possibly with multiplicity exceeding 1 .

One then has

$$
P L=\pi-Q D^{p}+R
$$

where $R \in C_{\mathrm{l}}[x][D], R=\sum b_{j} D^{j}$ with ord $R \leq p+l-1, \operatorname{deg}\left(b_{j}\right) \leq \sigma_{2} p$ (with $\sigma_{2}$ depending on $L$ but not on $\left.\mathfrak{p}\right),\left|b_{j}\right|_{\mathfrak{p}} \leq 1 / p^{f(\mathfrak{p})}$ (with $f(\mathfrak{p})=\left[\bar{K}_{\mathfrak{p}}: \boldsymbol{F}_{p}\right]$ ), where $\left|b_{j}\right|_{p}$ denotes the Gauss norm of $b_{j}$.

For $0<r<1$, let $B_{r}:=\left\{x \in C_{p} ;|x| \leq r^{-1},\left|x-c_{j}\right| \geq r\right\}$ where $c_{j}$ runs through the zeroes of $\pi$ and let $H=H\left(B_{r}\right)$ be the space of analytic elements on $B_{r}$ equipped with the sup norm \|\|$_{H}$ on $B_{r}$.

Then $\pi$ is invertible on $B_{r}$ and as $\operatorname{deg} \pi \leq \sigma_{1} p$, and as the gauss norm $|\pi|_{\mathfrak{p}}$ of $\pi$ is 1 , one obtains

$$
\|1 / \pi\|_{H} \leq 1 / r^{\sigma_{1} p}
$$

Using the estimates on the order and on the degrees of the coefficients of $P$, $Q, R$, we also obtain the estimates of the operator norms of $P, Q, R$ and $D^{p}$ 
considered as endomorphisms of $H$

$$
\begin{aligned}
& \left\|D^{p}\right\|_{H} \leq|p|_{\mathfrak{p}} / r^{p} \\
& \|Q\|_{H} \leq 1 / r^{\sigma_{1} p+l-1} \\
& \|R\|_{H} \leq 1 /\left(p^{f(\mathfrak{p})} r^{\sigma_{2} p+p+l-1}\right) .
\end{aligned}
$$

Finally we have

$$
\left\|\pi^{-1}\left(-Q D^{p}+R\right)\right\|_{H} \leq 1 /\left(p r^{\sigma_{3} p+l-1}\right)
$$

where $\sigma_{3}$ and $\omega$ depend on $L$ but not on $\mathfrak{p}$.

Therefore if $r=r_{p}:=1 /\left(p^{1 / 2\left(\sigma_{3} p+l-1\right)}\right)$, we have

$$
\left\|\pi^{-1}\left(-Q D^{p}+R\right)\right\|_{H}<1,
$$

which shows that $\pi^{-1} P L=1+\pi^{-1}\left(-Q D^{p}+R\right)$ is invertible in $H$ and so is $P L$.

Consider a disk $\Delta$ of radius $r=r_{\mathfrak{p}}$ contained in $B_{r}$. The same estimates hold on $H(\Delta)$ and therefore again $P L$ is invertible in $H(\Delta)$. Thus $P$ is surjective in $H(\Delta)$ and by Lemma 2.3 we see that $P$ is injective in $H(\Delta)$ and therefore $P$ is also injective in $H\left(B_{r}\right)$ as $H\left(B_{r}\right) \subset H(\Delta)$.

Thus $P$ is surjective and injective in $H$, and as $P L$ is invertible, we conclude that $L$ is invertible in $H$. Therefore the equation $L v=\varphi$ has a unique solution $v \in H$.

As $\mathfrak{p} \notin P(K)$, for all $n\left|u_{n}\right|_{\mathfrak{p}} \leq 1$, and $u$ defines a bounded analytic function in the unit open ball $B^{-}(0,1)$ of $C_{\mathfrak{p}}$.

Let $\Delta_{1}$ be the annulus $B_{r_{\mathrm{p}}} \cap B^{-}(0,1)$ and let $W$ be the space of functions analytic and bounded on $\Delta_{1}$. The same estimates hold and the same argument can be used to prove that $L$ is invertible in $W$. The restrictions of $u$ and $v$ to $\Delta_{1}$ are both in $W$ and both solutions of $L v=\varphi$, therefore these restrictions coincide, which means that $v$ is the analytic extension of $u$ on $B_{r_{p}}$.

But now that we know that $u$ is an analytic element on $B^{-}(0,1)$, we can use Lemma 2.5 to conclude that $u$ extends analytically in all the residue classes except in at most $M$ of them, with $M=\operatorname{ord} L+m(L)$.

Finally we have shown that $u$ extends analytically onto a set of the form

$$
\left\{x \in C_{\mathfrak{p}} ;|x| \leq 1 / r_{\mathfrak{p}},\left|x-c_{j}\right| \geq r_{\mathfrak{p}}, 1 \leq j \leq \mathscr{P}_{\mathfrak{p}}\right\}
$$

where the $c_{j}$ are in distinct residue classes and $\mathscr{P}_{\mathfrak{p}} \leq M$.

It is equivalent to say that $f$ defines in $C_{\mathfrak{p}}$ an analytic function on a set whose complementary is the union of $\mathscr{P}_{\mathfrak{p}}+1$ disks of radii $r_{\mathfrak{p}}$ contained in distinct residue classes and therefore of transfinite diameter $d_{\mathfrak{p}}=r_{\mathfrak{p}}^{1 /\left(\mathscr{P}_{\mathfrak{p}}+1\right)}$.

(v) The result of (iv) is valid for all prime ideals $p$ of a set $S$ satisfying condition (1.7.1). Therefore

$$
\prod_{\mathfrak{p} \in S} d_{\mathfrak{p}}=\prod_{\mathfrak{p} \in S} r_{\mathfrak{p}}^{1 /\left(\mathscr{P}_{\mathfrak{p}}+1\right)} \leq \prod_{\mathfrak{p} \in S} r_{\mathfrak{p}}^{1 /(M+1)}=0
$$


because

$$
\sum_{\mathfrak{p} \in S} \frac{1}{M+1} \log r_{\mathfrak{p}}=-\sum_{\mathfrak{p} \in S, \mathfrak{p} \mid p} \frac{1}{2(M+1)\left(\sigma_{3} p+l-1\right)} \log p=-\infty
$$

One can then find a finite subset $S_{1}$ of $S$ such that

$$
\prod_{w \in I(K)} d_{w}^{N(w)} \prod_{\mathfrak{p} \in P(K)} d_{\mathfrak{p}} \prod_{\mathfrak{p} \in S_{1}} d_{\mathfrak{p}}<1
$$

One then applies the rationality criterion 2.2 , with $\mathscr{P}_{1}(K)=P(K) \cup S_{1}$, to conclude that $f$, and therefore $u$, is rational.

\section{Operators of order one}

We shall now discuss the different possibilities for an operator or order 1 , $L=D+A$, with $A \in K(x)$.

(i) 0 is an irregular singularity of $L$, therefore a totally irregular singular point of $L$, and by Proposition $1.9 L$ is not a Pólya operator.

(ii) 0 is not an irregular singular point of $L$, but there exists $a \in K^{\text {alg }} \cup\{\infty\}$ which is an irregular singular point of $L$ and therefore a totally irregular singular point of $L$. Then by Theorem 1.7 and Proposition 1.11 we conclude that $L$ is a Pólya operator.

(iii) $L$ does not have irregular singular points. Then $A(x)=\sum_{i} \alpha_{i} /\left(x-a_{i}\right)$. The singular points of $L$ are the $a_{i}$ with exponent $\alpha_{i}$. If one of the exponents $\alpha_{i}$ is not rational, we can again apply Theorem 1.7 and the argument of 1.12 to conclude that $L$ is a Pólya operator.

(iv) There remains the case when $A(x)=\sum_{i} \alpha_{i} /\left(x-a_{i}\right)$ with $\alpha_{i} \in \mathbb{Q}$ for all $i$. Observe that for all $n \in \mathbf{Z}$ and all $a \in K^{\text {alg }}(x-a)^{n} L(x-a)^{-n}=L-n /(x-a)$ is equivalent to $L$. Thus by considering an equivalent operator we can reduce to the case where all the $\alpha_{i} \in \mathbf{Q}-\mathbf{Z}$. The different situations are

$L=D ;$ this is a Pólya operator (Theorem 1.2),

$L=D-\alpha / x ;$ this is a Pólya operator (Theorem 1.3),

$L=D-\sum_{i=1}^{s} \alpha_{i} /\left(x-a_{i}\right)$ with $s \geq 1, \alpha_{i} \in \mathbf{Q}-\mathbf{Z}, a_{i} \neq 0$. Then $u=$ $\prod_{i}\left(1-x / a_{i}\right)^{\alpha_{i}} \in \tilde{K}[[x]]$, is solution of $L u=0$ and is not rational. So $L$ is not a Pólya operator.

$L=D-\alpha / x-\sum_{i=1}^{s} \alpha_{i} /\left(x-a_{i}\right)$ with $s \geq 1, \alpha, \alpha_{i} \in \mathbf{Q}-\mathbf{Z}, a_{i} \neq 0$. In this case we cannot prove either that $L$ is a Pólya operator or that it is not. 


\section{References}

[1] Y. Amice, Les nombres p-adiques (PUF Collection Sup 1977).

[2] J.-P. Bezivin, 'Une propriété arithmétique de certains opérateurs différentiels', Manuscripta Math. 57 (1987), 351-372.

[3] D. Cantor, 'On arithmetic properties of coefficients of rational function, Pacific J. Math. 15 (1965), 55-58.

[4] D. Clark, 'A note on the p-adic convergence of linear differential equations', Proc. Amer. Math. Soc. 17 (1966), 262-269.

[5] B. Dwork and P. Robba, 'On ordinary linear p-adic differential equations', Trans. Amer. Math. Soc. 231 (1977), 1-46.

[6] T. Honda, 'Algebraic differential equations', Symposia Math. 24 (1981), 169-204.

[7] G. Janusz, Algebraic number fields (Academic Press, New York, 1973).

[8] G. Polya, 'Arithmetische Eigenschaften der Reiherenwichlungen rationaler Funktionen', J. Reine Angew. Math. 151 (1921), 1-31.

[9] P. Robba, 'Prolongement des solutions d'une équations différentielle p-adique', C.R. Acad. Sci. Paris 279 (1974), 153-154.

[10] P. Robba, 'On the index of p-adic differential operators II,' Duke Math. J. 43 (1976), 19-31.

Université Paris VI Math.

Université Paris-Sud Math.

Tour 45-46, 5ème étage

Bâtiment 425

4 , place Jussieu

91405 Orsay Cedex

75230 Paris Cedex 05

France

France 